\begin{tabular}{|c|l|}
\hline Title & $\begin{array}{l}\text { Effect of the cluster magnetization on the magnetotransport at low temperatures in ordered arrays of MnA snanoclusters } \\
\text { on (111)B GaA s }\end{array}$ \\
\hline Author(s) & Elm, M. T.; Klar, P. J.; Ito, S.; Hara, S.; Krug von Nidda, H.-A. \\
\hline Citation & $\begin{array}{l}\text { Physical Review B, 84(3), 035309-1-035309-8 } \\
\text { https://doi.org/10.1103/PhysRevB.84.035309 }\end{array}$ \\
\hline Issue Date & 2011-07-25 \\
\hline Doc URL & http://hdl.handle.net/2115/46914 \\
\hline Type & article \\
\hline Note & O2011 A merican Physical Society \\
\hline File Information & PhysRevB.84.035309.pdf \\
\hline
\end{tabular}

Instructions for use 


\title{
Effect of the cluster magnetization on the magnetotransport at low temperatures in ordered arrays of MnAs nanoclusters on (111) B GaAs
}

\author{
M. T. Elm* and P. J. Klar \\ Institute of Experimental Physics I, Justus-Liebig University, Heinrich-Buff-Ring 16, D-35392 Giessen, Germany \\ S. Ito and S. Hara \\ Research Center for Integrated Quantum Electronics, Hokkaido University, Sapporo 060-8628, Japan \\ H.-A. Krug von Nidda \\ Experimentalphysik V, University of Augsburg, D-86159 Augsburg, Germany \\ (Received 1 May 2011; revised manuscript received 8 June 2011; published 25 July 2011)
}

\begin{abstract}
The angle-dependent behavior of the magnetoresistance of ordered arrays of nanoclusters is studied. The arrays consist of single elongated MnAs nanoclusters, which were deposited on the (111)B GaAs substrate by selective-area metal-organic vapor phase epitaxy, which yields control of the position on the substrate as well as of the shape of the nanoclusters. Ferromagnetic resonance measurements were carried out in order to investigate the magnetic anisotropy of the nanoclusters, which is strongly determined by the cluster shape. Angle-dependent magnetotransport measurements were performed at $T=15 \mathrm{~K}$. The magnetic field was rotated in three different geometries, one parallel and two perpendicular to the sample surface. At low magnetic fields the nanoclusters show nearly no influence on the transport through the matrix. However, at high magnetic fields the magnetization orientation of the nanoclusters affects the transport behavior of the sample due to the influence of the clusters' dipolar field on the electronic states in the matrix. The experimental results obtained can be understood qualitatively by considering not only the transport properties of the GaAs matrix but also an average magnetization of the MnAs nanoclusters, whose orientation is determined by the magnetic anisotropy.
\end{abstract}

DOI: 10.1103/PhysRevB.84.035309

PACS number(s): 81.16.-c, 72.20.My, 75.30.Gw, 75.75.-c

\section{INTRODUCTION}

The realization of magnetoelectronic devices is currently of great interest due to their extended functionalities such as the nonvolatility of stored information in magnetic random access memories. ${ }^{1}$ Promising material systems for such devices may be the semiconductor-ferromagnetic granular hybrid structures, which consist of ferromagnetic nanoclusters embedded in a paramagnetic matrix. These material systems have the advantage, that their properties can be tuned in a wide range due to the large number of degrees of freedom (e.g., the cluster shape, cluster size, or mean distance between the nanoclusters). Furthermore, they show magnetoresistance effects similar to the giant magnetoresistance effect (GMR) and tunneling magnetoresistance effect (TMR). ${ }^{2-5}$ Additionally, with the method of selective-area metal-organic vapor-phase epitaxy (SA-MOVPE) on prepatterned (111)B GaAs substrates it is possible to control the position, the size, and the shape of MnAs nanoclusters, ${ }^{7,8}$ which allows one to actively tune the hybrids' properties. ${ }^{6}$ In particular, the arrangement of the nanoclusters strongly influences the transport path through the matrix and therefore the occurring magnetoresistance effects. ${ }^{9,10}$

In this paper, we present the results of angle-dependent magnetotransport measurements of three ordered nanocluster arrays, which consist of elongated MnAs nanoclusters grown by SA-MOVPE on prepatterned substrates. As shown by several groups, ${ }^{11-13}$ angle-dependent transport measurements are a powerful tool to probe the magnetic anisotropy as well as the switching behavior of the magnetization in ferromagnetic (Ga,Mn)As alloys. In the case of the arrangements with nanoclusters investigated, not only the transport through the nanoclusters but mainly the transport through the paramagnetic matrix is measured. Nevertheless, due to the inhomogeneous stray field of the ferromagnetic nanoclusters, a rotation of the clusters' magnetization orientation also affects the electronic states in the matrix and therefore the angle dependence of the resistivity. In order to determine the magnetic anisotropy of the nanoclusters, ferromagnetic resonance measurements were performed. For a theoretical description of the angledependent magnetoresistance, general expressions for the resistivity are used, which can be derived from symmetry considerations for the GaAs:Mn matrix. A good qualitative agreement between theory and experiment can be achieved when considering not only the transport properties of the paramagnetic matrix but also the clusters' magnetization in terms of an average magnetization orientation of the nanoclusters. The obtained results clearly show a strong influence not only of the nanoclusters' arrangement but also of their magnetization orientation on the transport behavior of GaAs:Mn/MnAs hybrid structures. An investigation of the switching behavior of the nanoclusters' magnetization and its influence on the magnetoresistance is therefore essential in order to access the potential of such hybrids for new magnetoelectronic devices.

\section{EXPERIMENTAL DETAILS}

One arrangement of single elongated nanoclusters and two arrangements of cluster chains were grown by SA-MOVPE on prepatterned semi-insulating (111) $B$ GaAs substrates. The (111) $B$ orientation of the substrate guarantees the selfassembled growth of high quality MnAs nanoclusters because 
the substrate and the $c$ plane of the NiAs-type clusters are of the same symmetry. In order to control the position and the shape of the nanoclusters on the substrate, $\mathrm{a} \mathrm{SiO}_{2}$ mask layer was deposited on the GaAs surface by plasma sputtering. Initial mask openings were prepared by electron beam lithography followed by wet-chemical etching with buffered hydrofluoric acid. The initial mask openings had a length of $650 \mathrm{~nm}$ and a width of $150 \mathrm{~nm}$. The two arrangements with cluster chains were prepared by reducing the distance between the initial openings to $50 \mathrm{~nm}$, in order to achieve a merging of the nanoclusters during growth. Prior to the growth of the MnAs nanoclusters, an AlGaAs buffer layer was deposited in the openings. The grown nanoclusters have a length of about $690 \mathrm{~nm}$ and a width of about $290 \mathrm{~nm}$. A detailed description of the structuring process and the growth conditions for both layers can be found elsewhere. ${ }^{7,8,10}$ The growth process also results in a $p$-type doping of the semi-insulating (111) $B \mathrm{GaAs}$ substrate, because Mn diffuses into the matrix at the initial openings. ${ }^{14}$ In the matrix, $\mathrm{Mn}$ is incorporated on the cation side of the GaAs lattice, ${ }^{15,16}$ where it acts as an acceptor and causes a paramagnetic behavior of the matrix.

All ordered arrays of nanocluster were investigated by scanning electron microscopy (SEM) using a Hitachi S4100 electron microscope. For the SEM observations of the nanoclusters, all the samples were tilted by approximately $45^{\circ}$ about the [1ํㅣㄹ direction acting as a rotation axis in order to obtain high-contrast images of the single nanoclusters. The acceleration voltage was $30 \mathrm{kV}$.

Ferromagnetic resonance measurements (FMR) were carried out for the ensembles of nanoclusters and cluster chains in order to determine the orientation of the magnetic easy axes in the sample plane. The measurements were performed with a Bruker ELEXSYS E500 CW-spectrometer at $X$-band frequency $(v \approx 9.35 \mathrm{GHz})$ and at a temperature of $280 \mathrm{~K}$. The samples were glued on suprasil-quartz rods in such a way, that the sample was rotated in in-plane geometry resulting in a rotation of the external magnetic field in the (111) plane.

The magnetotransport measurements were performed at $15 \mathrm{~K}$ in external magnetic fields up to $10 \mathrm{~T}$; dc currents of $500 \mathrm{pA}$ were applied along the [ $\overline{1} \overline{1} 2]$ direction. For the angledependent measurements, the external magnetic field was rotated in three different geometries, which are schematically shown in Fig. 1. In the first geometry, the external magnetic field was rotated in the (110) plane. For a rotation angle of $\alpha=0^{\circ}$ the external magnetic field is oriented parallel

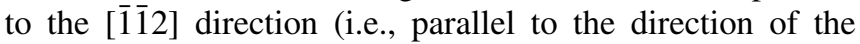
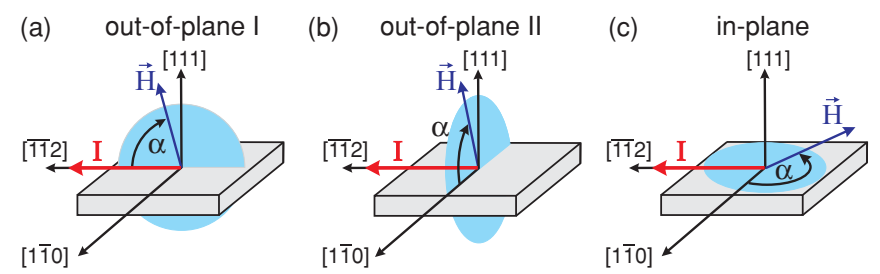

FIG. 1. (Color online) Used geometries for the angle-dependent measurements of the magnetoresistance. The external magnetic field was rotated (a) in the (110) plane for the out-of-plane I geometry, (b) in the (1112) plane for the out-of-plane II geometry, and (c) in the (111) plane for the in-plane geometry. applied current). In the following this geometry will be called out-of-plane I geometry. In the second geometry, the external magnetic field was rotated in the ( $1 \overline{1} 2)$ plane, where for $\alpha=0^{\circ}$ the field is oriented perpendicular to the current direction (i.e., along the [110] direction). This geometry is referred to as out-of-plane II geometry in the following. For the out-of-plane geometries the external magnetic field was rotated between $\alpha=-15^{\circ}$ and $175^{\circ}$. For the third geometry, the external magnetic field was rotated in the sample plane (i.e., in the (111) plane). For all in-plane measurements performed the external field was rotated between $-170^{\circ}$ and $120^{\circ}$.

\section{THEORETICAL BACKGROUND}

For a theoretical description of the angle-dependent magnetoresistance, we first introduce three unit vectors $\vec{k}, \vec{n}$, and $\vec{t}$, where $\vec{k}$ is parallel to the current direction, $\vec{n}$ is parallel to the surface normal, and $\vec{t}$ is defined by $\vec{t}=\vec{k} \times \vec{n}$. With these definitions and using Ohm's law,

$$
\vec{E}=\bar{\rho}(B) \cdot \vec{j},
$$

where $\vec{E}$ is the electric field, $\bar{\rho}$ is the resistivity tensor, and $\vec{j}$ is the current density, the magnetoresistivity $\rho_{\mathrm{MR}}$ can be written as

$$
\rho_{\mathrm{MR}}=\frac{E_{\mathrm{long}}}{j}=\vec{k} \cdot \bar{\rho}(B) \cdot \vec{k},
$$

where $E_{\text {long }}=\vec{k} \cdot \vec{E}$ is the component of the electric field parallel to the applied current direction $\vec{j}=j \cdot \vec{k}$. The resistivity tensor depends on the direction of the magnetic field. Thus, following the ansatz of Birss ${ }^{17}$ and Muduli et al., ${ }^{18} \bar{\rho}$ can be written in a series expansion in powers of $B_{i}$ :

$$
\rho_{i j}(B)=a_{i j}+a_{i j k} B_{k}+a_{i j k l} B_{k} B_{l}+\cdots,
$$

where the Einstein summation convention is used. For ferromagnetic materials and at low external magnetic fields, where the external field is much smaller than the sample's magnetization (i.e., $\left|\mu_{0} \vec{H}\right| \ll|\vec{M}|$ ), the magnetic flux density $\vec{B}=\mu_{0}(\vec{H}+\vec{M})$ can be replaced by only the contribution of the magnetization. ${ }^{12,13}$ However, in the case of the cluster arrangements investigated, the contribution of the external magnetic field $\mu_{0} \vec{H}$ on the paramagnetic matrix cannot be neglected, because the transport properties are mainly determined by the behavior of the matrix and only a small contribution arises from the influence of the nanoclusters' magnetization. For zincblende (Ga,Mn)As, one would assume that a theoretical description of the angular-dependent resistivity can be achieved using cubic symmetry $T_{\mathrm{d}}$. However, in the case of ferromagnetic (Ga,Mn)As grown on (001) and (311) $A$ substrates the angular dependence of the resistivity can be described successfully only by assuming cubic symmetry with tetragonal distortion along the [001] direction. ${ }^{12,13}$ This tetragonal distortion likely arises from compressive strain in the (Ga,Mn)As layer. ${ }^{12,19,20}$ Also in the case of the three arrays of nanoclusters and cluster chains grown on (111) $B$ substrates, a satisfying description of the experimental results can only be achieved by assuming hexagonal symmetry $\mathrm{C}_{6 \mathrm{~h}}$. Like for 
the $(\mathrm{Ga}, \mathrm{Mn})$ As alloys the hexagonal symmetry may arise due to a lattice distortion along the growth direction (i.e., along the [111] direction), caused by the incorporation of Mn into the matrix. Following the definition of the cubic coordinate system used by Smith et al. and considering only terms up to second order, the resistivity tensor representing the hexagonal symmetry can be written as ${ }^{21}$

$$
\begin{aligned}
\rho_{i j}(B)= & a_{11}\left(\begin{array}{ccc}
1 & 0 & 0 \\
0 & 1 & 0 \\
0 & 0 & 0
\end{array}\right)+a_{33}\left(\begin{array}{lll}
0 & 0 & 0 \\
0 & 0 & 0 \\
0 & 0 & 1
\end{array}\right) \\
& +a_{123}\left(\begin{array}{ccc}
0 & B_{z} & 0 \\
-B_{z} & 0 & 0 \\
0 & 0 & 0
\end{array}\right)+a_{1111}\left(\begin{array}{ccc}
B_{x}^{2} & 0 & 0 \\
0 & B_{y}^{2} & 0 \\
0 & 0 & 0
\end{array}\right) \\
& +a_{3333}\left(\begin{array}{ccc}
0 & 0 & 0 \\
0 & 0 & 0 \\
0 & 0 & B_{z}^{2}
\end{array}\right)+a_{2211}\left(\begin{array}{ccc}
B_{y}^{2} & 0 & 0 \\
0 & B_{x}^{2} & 0 \\
0 & 0 & 0
\end{array}\right) \\
& +a_{3311}\left(\begin{array}{ccc}
0 & 0 & 0 \\
0 & 0 & 0 \\
0 & 0 & B_{x}^{2}+B_{y}^{2}
\end{array}\right)+a_{1133}\left(\begin{array}{ccc}
B_{z}^{2} & 0 & 0 \\
0 & B_{z}^{2} & 0 \\
0 & 0 & 0
\end{array}\right) \\
& +a_{1112}\left(\begin{array}{ccc}
B_{x} B_{y} & 0 & 0 \\
0 & -B_{x} B_{y} & 0 \\
0 & 0 & 0
\end{array}\right) \\
& +a_{1212}\left(\begin{array}{ccc}
0 & B_{x} B_{y} & 0 \\
B_{x} B_{y} & 0 & 0 \\
0 & 0 & 0
\end{array}\right) .
\end{aligned}
$$

The orientation of the coordinate system with respect to the $a$ and $c$ axes of the hexagonal system is shown in Fig. 2(a). The direction of the unit vectors $\vec{k}, \vec{n}$, and $\vec{t}$ with respect to the crystal directions of the (111)B GaAs substrate are shown in Fig. 2(b). For the angle-dependent transport measurements, the current was applied along the [1112] direction, which corresponds to the $[0 \overline{1} 0]$ direction in the new coordinate system. Using Eqs. (2) and (4) the magnetoresistivity can be written as

$$
\rho_{\mathrm{MR}}=a_{11}+a_{2211} B_{x}^{2}+a_{1111} B_{y}^{2}+a_{1133} B_{z}^{2}-a_{1112} B_{x} B_{y} .
$$

As can be seen from Eq. (5) the magnetoresistivity strongly depends on the orientation of the magnetic flux density $\vec{B}=$ $\mu_{0}(\vec{H}+\vec{M})$. For a calculation of the resistivity it is therefore necessary to determine the orientation of the magnetization in dependence on the orientation of the external magnetic field $\mu_{0} H$.

\section{RESULTS AND DISCUSSION}

Scanning electron microscopy (SEM) images of all prepared arrangements of nanoclusters are shown in Fig. 3. The first regular array, shown in Fig. 3(a), consists of single elongated nanoclusters, which are oriented along the [110] direction. For the first arrangement with cluster chains, which is shown in Fig. 3(b), the distance between the elongated
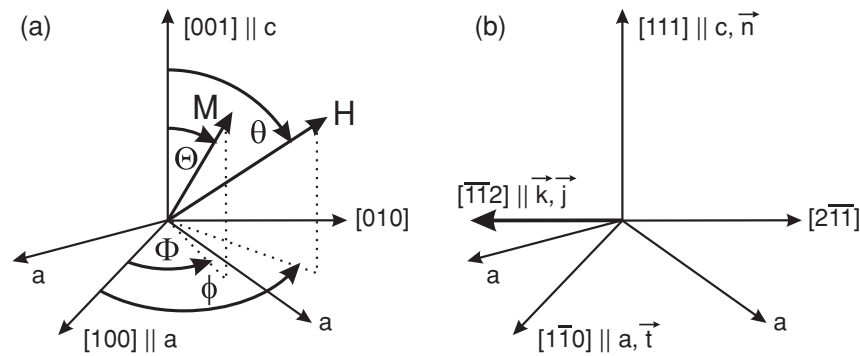

FIG. 2. (a) Definition of the used cubic coordinate system with respect to the $a$ and $c$ axes of the hexagonal crystal structure. (b) Definition of the unit vectors $\vec{k}, \vec{n}$, and $\vec{t}$ with respect to the crystal axes of the (111)B GaAs substrate. The azimuthal and polar orientation angles of the magnetization are denoted with $\Phi$ and $\Theta$, respectively.

nanoclusters was reduced to form cluster chains. The resulting chains are also oriented along the [1ㅣㅣ direction (i.e., perpendicular to the direction of the applied current). In the following, this arrangement is referred to as cluster chains I. The second cluster chain arrangement prepared consists of an alternating sequence of elongated nanoclusters, which

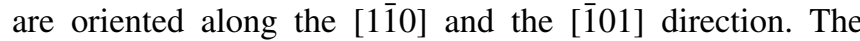
resulting cluster chains are oriented between the [101] and the [211] direction. This arrangement will be called cluster chains II in the following. The elongated nanoclusters of all three arrangements exhibit a high crystal quality with well-defined facets. But, only for the cluster chains I a full merging of the nanoclusters was achieved during the growth process, while small gaps between the single nanoclusters are observable for the cluster chains II.

In order to determine the magnetic anisotropy of the single elongated nanoclusters and the cluster chains FMR measurements were performed at $280 \mathrm{~K}$. Due to the rather weak resonance signal, measurements at lower temperatures were not possible. In out-of-plane geometry (not shown here) all three arrays with nanoclusters show a strong twofold anisotropy with a hard magnetic axis oriented along the [111]
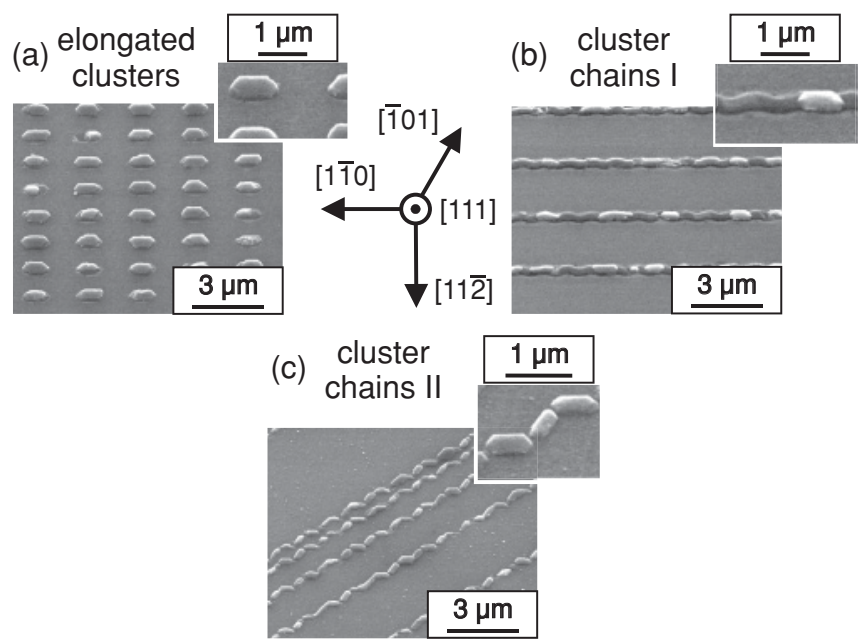

FIG. 3. Scanning electron microscopy images of the (a) arrangement with single elongated nanoclusters, (b) the arrangement with cluster chains I, and (c) the arrangement with cluster chains II, respectively. 

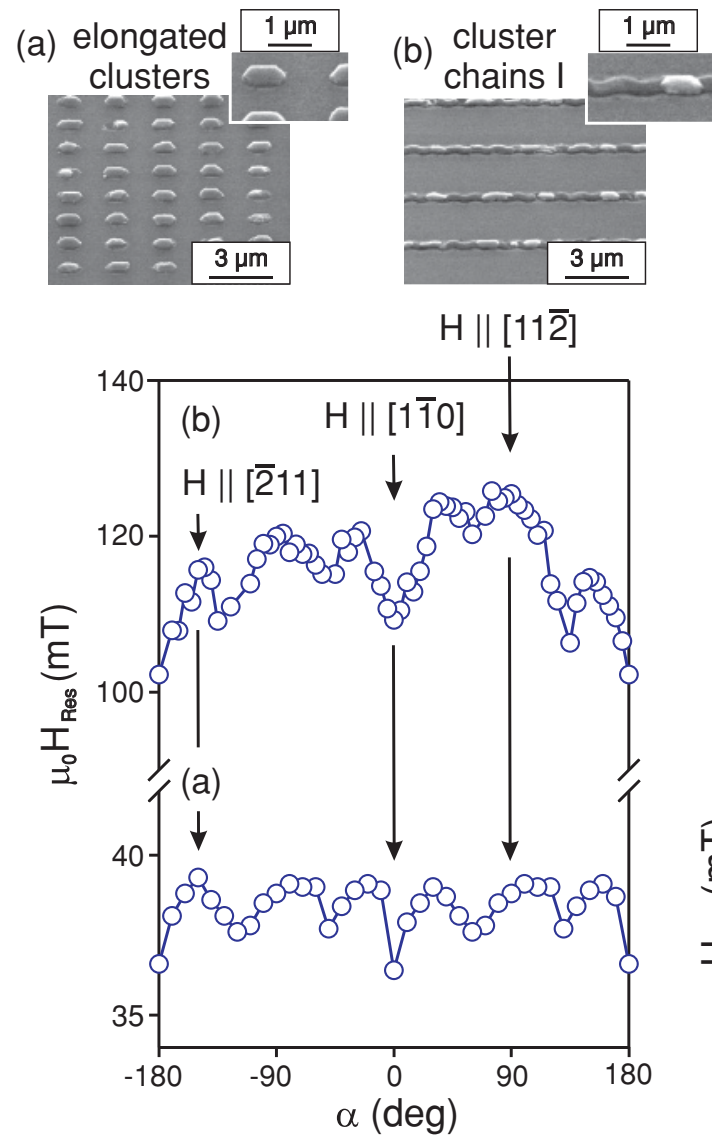
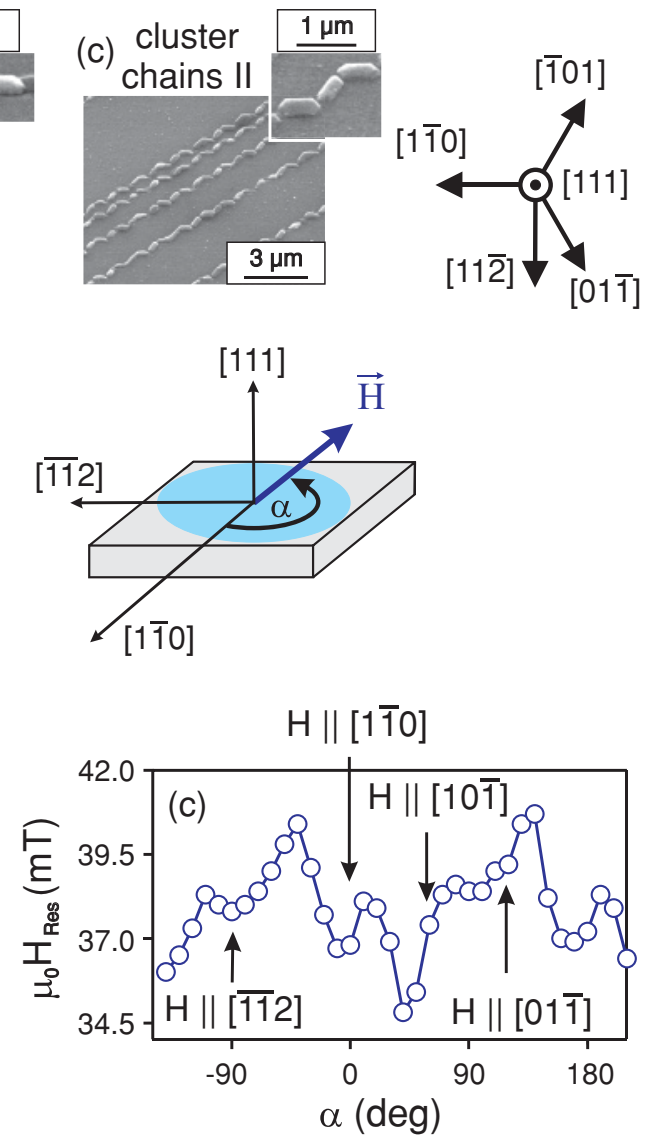

FIG. 4. (Color online) Ferromagnetic resonance measurements in in-plane geometry for all cluster arrangements investigated.

direction (i.e., parallel to the $c$ axes of the nanoclusters). An orientation of the magnetization in the cluster plane was also found by Hara et al., who performed angle-dependent measurements of the magnetization for randomly distributed nanoclusters on (111) $B$ GaInAs/InP surfaces. ${ }^{22}$

For all three arrangements FMR measurements in in-plane geometry were also performed. The results are shown in Fig. 4. Hexagon-shaped nanoclusters show a sixfold symmetry in the cluster plane corresponding to the hexagonal crystal symmetry and the cluster shape. ${ }^{10,22}$ Minima occur approximately along the $\langle 1 \overline{1} 0\rangle$ directions (i.e., parallel to the $a$ axes of the hexagon-shaped nanoclusters), which are easy axes of magnetization. ${ }^{23,24}$ For the elongated nanoclusters, the sixfold symmetry is overlaid by an additional weak $180^{\circ}$ anisotropy, which slightly modifies the minima. As a consequence the resonance signal exhibits two global minima in the sample plane, when the field is parallel to the [110] direction. The main easy axis of magnetization is oriented along this direction. Thus, the asymmetric shape of the nanoclusters forces the magnetization along the direction of elongation. For the arrangement with cluster chains I the same behavior can be observed as shown in Fig. 4(b). But compared to the single elongated nanoclusters the additional twofold anisotropy is much more pronounced. The formation of cluster chains due to the merging of the nanoclusters during the growth therefore increases the tendency of the magnetization to align along the orientation direction of the cluster chains. Additionally the twofold anisotropy modifies the position of the local minima, which are slightly shifted toward the nanoclusters' main axis of elongation. A similar behavior can also be found for the arrangement with cluster chains II. Although the nanoclusters did not merge during the growth, the small distances between the clusters enable them to couple. As shown in Fig. 4(c) this coupling results in an easy axis of magnetization oriented between the $[2 \overline{1} \overline{1}]$ and the $[10 \overline{1}]$ direction (i.e., approximately along the direction of the cluster chains) and a hard axis perpendicular to it.

Angle-dependent measurements of the magnetoresistance were performed at external magnetic fields of 1 and $10 \mathrm{~T}$, in order to investigate the influence of the ferromagnetic nanoclusters on the transport in the matrix. For both out-ofplane geometries, all nanocluster arrangements investigated show the same qualitative behavior for a given external magnetic field. The samples only exhibit different magnitudes of the resistance effects. Figure 5(a) shows exemplarily the results of the arrangement with cluster chains I for both out-of-plane geometries. At an external magnetic field of $\mu_{0} H=1 \mathrm{~T}$, shown in the top image of Fig. 5(a), a sine dependence of the resistance is observed, if the external magnetic field is rotated in the (110) plane (i.e., for the out-of-plane I geometry). In out-of-plane II geometry, the resistance shows an additional sharp decrease in the vicinity of $\alpha=0^{\circ}$, where the magnetic field is oriented parallel to the axis of elongation of the nanoclusters. At a high magnetic field of $\mu_{0} H=10 \mathrm{~T}$, also for the out-of-plane II geometry the resistance shows a deviation from the sine dependence, if the 

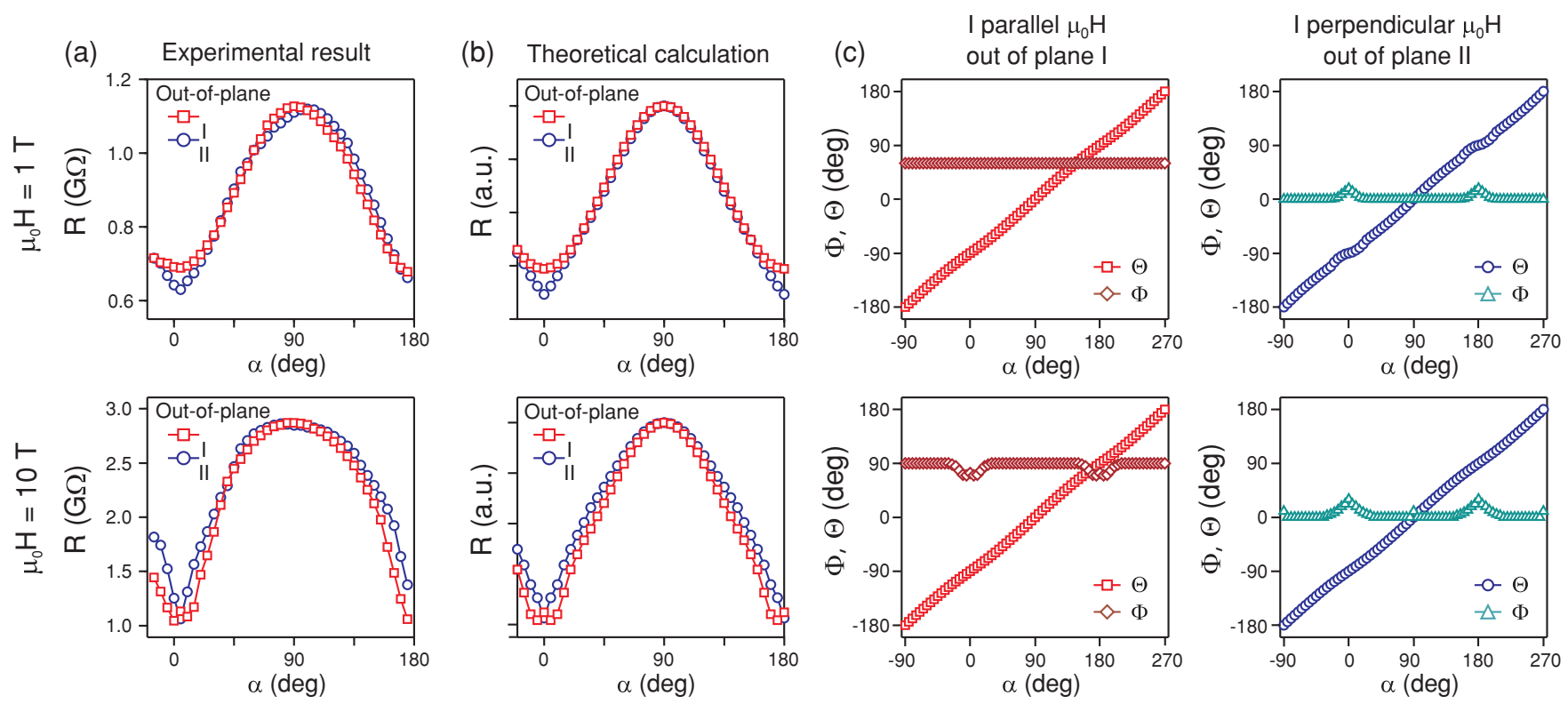

FIG. 5. (Color online) (a) Results of the angle-dependent transport measurements for the arrangement with cluster chains I in out-of-plane geometry for an external magnetic field of $1 \mathrm{~T}$ (top) and $10 \mathrm{~T}$ (bottom), respectively. (b) Corresponding theoretical calculations of the magnetoresistance behavior. (c) Magnetization angles $\Theta$ and $\Phi$ for a rotation of the magnetic field in the out-of-plane I and the out-of-plane II geometry, respectively.

magnetic field is oriented in the sample plane. Additionally, for both geometries a broadening of the maximum of the resistance is observed (i.e., for an orientation of the magnetic field parallel to the hard axis of the nanoclusters).

In order to explain this behavior and to determine the influence of the nanoclusters' magnetization on the transport properties, Eq. (5) was used for simulating the angle-dependent measurements. While the orientation of the external magnetic field was determined by the measurement geometry, the contribution of the magnetization to Eq. (5) was estimated by an average magnetization orientation of the arrangement of nanoclusters taking into account the results of the FMR measurements performed at $280 \mathrm{~K}$. The resistivity parameters were used as fitting parameters, which were varied to match the results of all three investigated geometries simultaneously. Thus, only a qualitative description of the angular dependence of the resistivity is presented.

The results of the calculations for both out-of-plane geometries are presented in Fig. 5(b). Figure 5(c) shows the corresponding angles $\Theta$ and $\Phi$ describing the orientation of the magnetization used for the calculations. At an external magnetic field of $1 \mathrm{~T}$ and in out-of-plane I geometry, a good qualitative agreement between theory and experiment can be achieved for a magnetization orientation

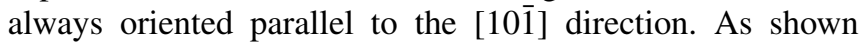
by the FMR measurements in Fig. 4(a) the nanoclusters exhibit one of the easy axes of magnetization along this direction, while the direction parallel to the external magnetic field is an in-plane hard magnetic axis. At low fields, the magnetic anisotropy therefore forces the magnetization along an easy axis leading to the observed sine dependence of the resistance.

For out-of-plane II geometry, the situation is different. For $\alpha=0^{\circ}$ the external magnetic field is oriented along the [1 $\left.\overline{1} 0\right]$ direction (i.e., parallel to the main easy axis of magnetization). However, a good qualitative agreement between theory and experiment can only be achieved, if the average cluster magnetization slightly rotates away from the main axis of elongation of the nanoclusters for an orientation of the external magnetic field near the sample plane. This behavior can be explained, on the basis of the FMR measurements. Because the additional twofold anisotropy due to the asymmetric cluster shape is relatively weak, a fraction of the nanoclusters may still exhibit a magnetization orientation along the easy axis parallel to the $[10 \overline{1}]$ direction. Thus, the average magnetization orientation slightly differs from the major elongation axis of the nanoclusters leading to a decrease of the resistance, if the external field rotates into the sample plane.

At a large magnetic field of $\mu_{0} H=10 \mathrm{~T}$ for out-of-plane II geometry a behavior of the magnetization similar to that at low magnetic fields is observed, if the external magnetic field is oriented in the sample plane. Again the average magnetization seems to rotate away from the major cluster axis, that is, even at high magnetic fields a fraction of nanoclusters still exhibits a magnetization oriented along the local minima parallel to the [101] direction. Also for the out-of-plane I geometry the average magnetization is not oriented parallel to the external magnetic field direction in the sample plane, but between

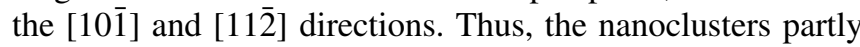
exhibit a magnetization orientation along the easy axis of magnetization. However, for a rotation out of the sample plane, the magnetization of the nanoclusters aligns parallel to the external magnetic field direction. In addition, the tendency of a broadening of the resistance maximum can be observed in the theoretical results. However, the pronounced broadening of the experimental results cannot be reproduced entirely using Eq. (5). At a magnetic field of $10 \mathrm{~T}$ the arrangements exhibit large relative magnetoresistance effects up to $900 \%{ }^{14}$ 

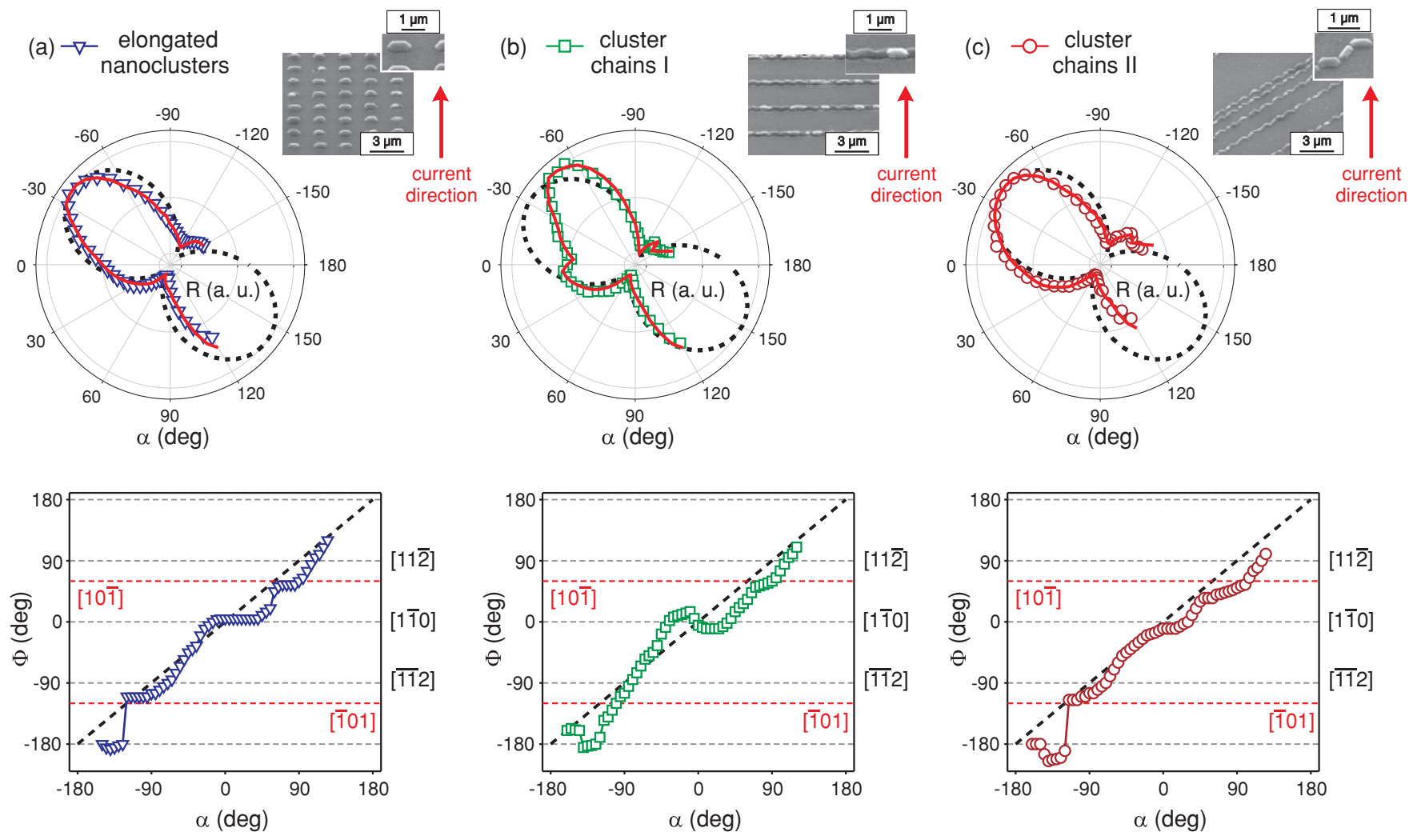

FIG. 6. (Color online) (Top) Polar plot of the angular dependence of the resistance at $10 \mathrm{~T}$ in in-plane geometry for the arrangements with (a) single elongated nanoclusters, (b) cluster chains I, and (c) cluster chains II. The black dashed line represents the angle-dependent resistance of the paramagnetic matrix, where the influence of the nanoclusters is neglected. The red solid line shows the calculated resistance curves. (Bottom) Corresponding in-plane angle $\Phi$ of the magnetization used for the calculations.

Therefore, the angular dependence of the resistance in out-ofplane geometry may not be described in the low-field limit or terms of higher order have to be considered in Eq. (5).

Also for the angle-dependent measurements in in-plane geometry all three cluster arrangements investigated show a similar behavior at a low magnetic field of $\mu_{0} H=1 \mathrm{~T}$. For a rotation of the external magnetic field in the sample plane the transport behavior of all three arrangements is mainly determined by the behavior of the paramagnetic matrix leading to the ordinary sine dependence of the resistance (not shown here). However, at a high magnetic field of $10 \mathrm{~T}$ the three cluster arrangements exhibit differences in the angular dependence of the resistance. For a better comparison of the three arrangements the resistance was normalized to values between 1 and 2. The results are shown in Fig. 6 in a polar representation.

Figure 6(a) shows the angular dependence of the resistance for the arrangement with single elongated nanoclusters. The calculated behavior of the paramagnetic matrix (i.e., where the influence of the ferromagnetic nanoclusters on the transport properties is neglected) is also shown as a black dashed line. Compared to the sine-dependent behavior of the matrix without clusters the resistance of the hybrid arrangement with elongated nanoclusters is increased between $\alpha=-150^{\circ}$ and $-120^{\circ}$. Also along the main axis of cluster elongation (i.e., between $-30^{\circ}$ and $30^{\circ}$ ), the resistance exhibits slight deviations from the sine-dependent behavior. Additionally, between $\alpha=-90^{\circ}$ and $-60^{\circ}$ as well as between $90^{\circ}$ and $120^{\circ}$ the resistance is decreased compared to the assumed behavior of the pure paramagnetic matrix. A similar behavior is also observed for the arrangement with cluster chains I [Fig. 6(b)]. For this arrangement the deviations in the resistance for an angle of rotation between $-45^{\circ}$ and $30^{\circ}$ are even more pronounced than for the arrangement with single elongated nanoclusters. Moreover, the resistance exhibits a local minimum for an orientation of the external magnetic field close to the [1ㅣㅣㄹ direction (i.e., along the main axes of elongation of the cluster chains). The arrangement with cluster chains II, shown in Fig. 6(c), mainly exhibits the expected sine dependence of the resistance. Only between $\alpha=-90^{\circ}$ and $-60^{\circ}$ as well as between $90^{\circ}$ and $120^{\circ}$ the resistance of the arrangement is decreased compared to the calculated matrix behavior. Like for the other two arrangements, the arrangement with cluster chains II also exhibits an increase of the resistance between $-150^{\circ}$ and $-120^{\circ}$.

As for the results in out-of-plane geometry, a comparison between the experimental results in in-plane geometry and the calculated behavior of the paramagnetic matrix shows that the influence of the ferromagnetic nanoclusters on the transport properties cannot be neglected. Thus, again Eq. (5) was used for describing the experimental results. The calculated resistance curves, for which the best agreement between theory and experiment was achieved, are shown in Fig. 6 as red solid lines. The corresponding in-plane angle $\Phi$ of the magnetization used for the calculations is presented in the bottom of Fig. 6, respectively. A comparison of the magnetization orientation 
of all three cluster arrays shows that the increase of the resistance for an angle of rotation between $-150^{\circ}$ and $-120^{\circ}$ originates from an abidance of the magnetization close to the [110] direction, that is, along the major easy axis of magnetization of the nanoclusters. For the arrangement with single elongated nanoclusters the magnetization jumps into an orientation parallel to the [101] direction, if the external field reaches an angle of rotation of $-120^{\circ}$. As shown by the FMR measurements in Fig. 4(a) this direction is also an easy axis of magnetization. Again the magnetization remains parallel to this direction until the external field reaches $\alpha=-80^{\circ}$. The magnetization then starts to rotate toward the main axis of elongation parallel to the [110] direction (i.e., the main easy axis of magnetization). There it stays until it jumps directly into the neighbored easy axis parallel to the [101] direction. This steplike rotation of the magnetization with the abidance close to the easy axes of magnetization of the nanoclusters causes the observed deviations of the angledependent resistance compared to the pure matrix behavior. The average magnetization of the arrangement with cluster chains I shows a similar behavior. However, due to the stronger twofold anisotropy caused by the cluster shape as shown in Fig. 4(b), the magnetization mainly abides close to the main axes of the cluster chains, where the resonance of the FMR signal shows global minima. For $\alpha>-120^{\circ}$ it directly rotates to an orientation parallel to the [1 $1 \overline{10}]$ direction and does not jump into the weaker easy axis parallel to the [101] direction as in the case of the single elongated nanoclusters. Near the [1ㅣㅣㄹ direction the magnetization orientation fluctuates between $\Phi=-15^{\circ}$ and $15^{\circ}$. This behavior confirms the assumptions made for describing the results for out-of-plane II geometry, where the average magnetization orientation was supposed to slightly deviate from the [110] direction, when the external field is oriented parallel to this direction. At $\alpha=40^{\circ}$ the magnetization rotates toward the [101] direction, where it shortly remains and finally, the magnetization orientation begins to rotate again parallel to the external magnetic field direction at $\alpha=95^{\circ}$. The much stronger twofold anisotropy of the nanocluster chains oriented along the [1ㅣㅣ direction and the resulting magnetization behavior are therefore responsible for the occurrence of the local minimum in the resistance observed for the arrangement with cluster chains I. For the arrangement with cluster chains II the magnetization orientation is mainly parallel to the external magnetic field direction compared to the other two arrangements investigated. Small deviations occur at $\alpha=-180^{\circ}$ and $0^{\circ}$, where the magnetization again remains parallel to the cluster main axes oriented along the [110] direction. But a strong deviation of the magnetization behavior is observed for $\alpha=45^{\circ}$. Here the magnetization abides at about $\Phi=50^{\circ}$ (i.e., below the [101] direction), which is one easy axis of magnetization for these cluster chains, as shown by the FMR measurements in Fig. 4(c).

For all three cluster arrays the angle-dependent behavior of the magnetoresistance can therefore be described qualitatively only when the influence of the clusters' magnetization is accounted for. In contrast to the out-of-plane geometries, a satisfying description of all three arrangements was possible in the low field limit even at high magnetic fields. It seems that this is possible because the magnetoresistance effects in in-plane geometry are much smaller compared to the effects in out-of-plane geometry. Thus, the used low field limit still provides a good qualitative description of the experimental results observed.

\section{SUMMARY}

Three different ordered arrays of elongated nanoclusters and cluster chains were grown by SA-MOVPE. Ferromagnetic resonance measurements in the sample plane show an additional twofold anisotropy of the elongated nanoclusters due to their asymmetric shape, leading to a preferred orientation of the magnetization along the main axis of elongation of the single nanoclusters and cluster chains. Angle-dependent magnetoresistance measurements were performed in order to investigate the influence of the nanoclusters' magnetization on the transport properties. At low magnetic fields, the resistance shows a sine-dependent behavior as expected for the paramagnetic matrix. At high magnetic fields, in out-ofplane geometry as well as in in-plane geometry, the angular dependence of the resistance exhibits deviations from the predicted sine-dependent behavior of the pure paramagnetic matrix. In out-of-plane geometry a modeling of the resistance in the low field limit is not sufficient for describing the experimental results satisfactorily. However, the behavior in in-plane geometry, where the magnetoresistance effects are much smaller, can be described qualitatively by accounting for an average behavior of the nanoclusters' magnetization. Thus, the magnetization orientation of the nanoclusters has a direct influence on the transport properties of the paramagnetic matrix in such ordered hybrid structures, which cannot be neglected.

\section{ACKNOWLEDGMENTS}

We thank the German Research Foundation (DFG) and Japan Society for the Promotion of Science for financial support. M.T.E. thanks the Justus-Liebig University for providing his PhD scholarship. *matthias.elm@physik.uni-giessen.de

${ }^{1}$ G. A. Prinz, Science 282, 1660 (1998).

${ }^{2}$ A. E. Berkowitz, J. R. Mitchell, M. J. Carey, A. P. Young, S. Zhang, F. E. Spada, F. T. Parker, A. Hutten, and G. Thomas, Phys. Rev. Lett. 68, 3745 (1992).

${ }^{3}$ W. Heimbrodt, P. J. Klar, S. Ye, M. Lampalzer, C. Michel, S. D. Baranovskii, P. Thomas, and W. Stolz, J. Supercond. 18, 315 (2005).
${ }^{4}$ J. Q. Xiao, J. S. Jiang, and C. L. Chien, Phys. Rev. Lett. 68, 3749 (1992).

${ }^{5}$ P. J. Wellmann, J. M. Garcia, J.-L. Feng, and P. M. Petroff, Appl. Phys. Lett. 73, 3291 (1998).

${ }^{6}$ H.-A. Krug von Nidda, T. Kurz, A. Loidl, T. Hartmann, P. J. Klar, W. Heimbrodt, M. Lampalzer, K. Volz, and W. Stolz, J. Phys. Condens. Matter 18, 6071 (2006). 
${ }^{7}$ T. Wakatsuki, S. Hara, S. Ito, D. Kawamura, and T. Fukui, Jpn. J. Appl. Phys. 48, 04C137 (2009).

${ }^{8}$ S. Ito, S. Hara, T. Wakatsuki, and T. Fukui, Appl. Phys. Lett. 94, 243117 (2009).

${ }^{9}$ C. Michel, M. T. Elm, B. Goldlücke, S. D. Baranovskii, P. Thomas, W. Heimbrodt, and P. J. Klar, Appl. Phys. Lett. 92, 223119 (2008)

${ }^{10}$ M. T. Elm, C. Michel, J. Stehr, D. M. Hofmann, P. J. Klar, S. Ito, S. Hara, and H.-A. Krug von Nidda, J. Appl. Phys. 107, 013701 (2010).

${ }^{11}$ S. T. B. Goennenwein, S. Russo, A. F. Morpurgo, T. M. Klapwijk, W. Van Roy, and J. De Boeck, Phys. Rev. B 71, 193306 (2005).

${ }^{12}$ W. Limmer, M. Glunk, J. Daeubler, T. Hummel, W. Schoch, R. Sauer, C. Bihler, H. Huebl, M. S. Brandt, and S. T. B. Goennenwein, Phys. Rev. B 74, 205205 (2006).

${ }^{13}$ M. T. Elm, P. J. Klar, W. Heimbrodt, U. Wurstbauer, M. Reinwald, and W. Wegscheider, J. Appl. Phys. 103, 093710 (2008).

${ }^{14}$ M. T. Elm, P. J. Klar, S. Ito, and S. Hara, Phys. Rev. B 83, 235305 (2011).
${ }^{15}$ T. Jungwirth, J. Sinova, J. Mašek, J. Kučera, and A. H. MacDonald, Rev. Mod. Phys. 78, 809 (2006).

${ }^{16}$ R. Shioda, K. Ando, T. Hayashi, and M. Tanaka, Phys. Rev. B 58, 1100 (1998).

${ }^{17}$ R. R. Birss, Symmetry and Magnetism (North Holland, Amsterdam, 1966).

${ }^{18}$ P. K. Muduli, K.-J. Friedland, J. Herfort, H.-P. Schönherr, and K. H. Ploog, Phys. Rev. B 72, 104430 (2005).

${ }^{19}$ X. Liu, Y. Sasaki, and J. K. Furdyna, Phys. Rev. B 67, 205204 (2003).

${ }^{20}$ C. Bihler, H. Huebl, M. S. Brandt, S. T. B. Goennenwein, M. Reinwald, U. Wurstbauer, M. Doppe, D. Weiss, and W. Wegscheider, Appl. Phys. Lett. 89, 012507 (2006).

${ }^{21}$ A. C. Smith, J. F. Janak, and R. B. Adler, Electronic Conduction in Solids (McGraw-Hill, New York, 1967).

${ }^{22}$ S. Hara and T. Fukui, Appl. Phys. Lett. 89, 113111 (2006).

${ }^{23}$ K. Ando, A. Chiba, and H. Tanoue, Appl. Phys. Lett. 73, 387 (1998).

${ }^{24}$ K.-J. Friedland, M. Kästner, and L. Däweritz, J. Supercond. 16, 261 (2003). 